International Journal of Pure and Applied Mathematics

Volume 109 No. 1 2016, 29-48

ISSN: 1311-8080 (printed version); ISSN: 1314-3395 (on-line version)

url: http://www.ijpam.eu

doi: 10.12732/ijpam.v109i1.4

\title{
ON PERTURBED FRACTIONAL DIFFERENTIAL EQUATIONS AND INCLUSIONS WITH GENERALIZED RIEMANN-LIOUVILLE FRACTIONAL INTEGRAL BOUNDARY CONDITIONS
}

\author{
Bashir Ahmad ${ }^{1}$, Sotiris K. Ntouyas ${ }^{1,2}$ \\ ${ }^{1}$ Nonlinear Analysis and Applied Mathematics (NAAM)-Research Group \\ Department of Mathematics \\ Faculty of Science \\ King Abdulaziz University \\ P.O. Box 80203, Jeddah 21589, SAUDI ARABIA \\ ${ }^{2}$ Department of Mathematics \\ University of Ioannina \\ 451 10, Ioannina, GREECE \\ ${ }^{2}$ e-mail: sntouyas@uoi.gr
}

\begin{abstract}
In this paper, we present the sufficient criteria for the existence of solutions for perturbed fractional differential equations and inclusions with generalized Riemann-Liouville fractional integral boundary conditions. We make use of a nonlinear alternative, which deals with the sum of completely continuous and contractive single-valued or multi-valued operators, to obtain the desired results.
\end{abstract}

AMS Subject Classification: $34 \mathrm{~A} 08,34 \mathrm{~A} 12,34 \mathrm{~A} 60$

Key Words: Caputo fractional derivative, generalized Riemann-Liouville fractional integral, fractional differential inclusions, existence, fixed point theorems

Received: August 1, 2016

Published: September 1, 2016
(C) 2016 Academic Publications, Ltd. url: www.acadpubl.eu 


\section{In Memory of Drumi Bainov \\ July 2, 1933 - July 1, 2011.}

\section{Introduction}

The subject of fractional differential equations has emerged as an interesting and popular field of research in view of its extensive applications in applied and technical sciences. One can easily observe the role and importance of fractional calculus in several diverse disciplines such as physics, chemical processes, population dynamics, biotechnology, economics, etc. For examples and recent development on the topic, see [1]-[19] and the references cited therein. The significance of fractional derivatives owes to the fact that they serve as an excellent tool for the description of memory and hereditary properties of various materials and processes. One can notice that fractional derivatives are defined via fractional integrals. Among several types of fractional integrals found in the literature, Riemann-Liouville and Hadamard fractional integrals are the extensively studied ones. A new fractional integral, called generalized RiemannLiouville fractional integral, which generalizes the Riemann-Liouville and the Hadamard integrals into a single form, was introduced in [20] (see Definition 2.6). For more details of this integral and similar ones, we refer the reader to the text [21] and the papers [22, 23].

In this paper, we study perturbed boundary value problems of fractional differential equations and inclusions supplemented with generalized RiemannLiouville fractional integral conditions. In precise terms, we consider the singlevalued and multi-valued nonlocal problems respectively given by

$$
\left\{\begin{array}{l}
D^{\alpha} x(t)=f(t, x(t))+g(t, x(t)), \quad t \in[0, T], \\
x(0)=0, \\
x(T)=\beta \frac{\rho^{1-q}}{\Gamma(q)} \int_{0}^{\xi} \frac{s^{\rho-1}}{\left(\xi^{\rho}-s^{\rho}\right)^{1-q}} x(s) d s:=\beta^{\rho} I^{q} x(\xi), \quad 0<\xi<T,
\end{array}\right.
$$

and

$$
\left\{\begin{array}{l}
D^{\alpha} x(t) \in F(t, x(t))+G(t, x(t)), \quad t \in[0, T], \\
x(0)=0, \quad x(T)=\beta^{\rho} I^{q} x(\xi), \quad 0<\xi<T,
\end{array}\right.
$$

where $D^{\alpha}$ is the Caputo fractional derivative of order $1<\alpha \leq 2, f, g$ : $[0, T] \times \mathbb{R} \rightarrow \mathbb{R}$ are continuous functions, ${ }^{\rho} I^{q}$ is the generalized RiemannLiouville fractional integral of orders $q>0, \rho>0$, and $F, G:[0, T] \times \mathbb{R} \rightarrow \mathcal{P}(\mathbb{R})$ are multi-valued functions, $(\mathcal{P}(\mathbb{R})$ is the family of all nonempty subjects of $\mathbb{R})$. 
The paper is organized as follows: In Section 2 we present some preliminary concepts of fractional calculus and lemmas. Section 3 contains the existence result for problem (1) which is established via a fixed point theorem due to Burton and Kirk [24]. In Section 4, we discuss the existence of solutions for problem (2) by means of a nonlinear alternative for contractive maps [25].

\section{Preliminaries}

In this section, we introduce some notations and definitions of fractional calculus $[1,2]$ and present some results needed in the sequel.

Definition 2.1. The Riemann-Liouville fractional integral of order $q>0$ of a continuous function $f:(0, \infty) \rightarrow \mathbb{R}$ is defined by

$$
J^{q} f(t)=\frac{1}{\Gamma(q)} \int_{0}^{t}(t-s)^{q-1} f(s) d s,
$$

provided the right-hand side is point-wise defined on $(0, \infty)$ and $\Gamma$ is the gamma function given by $\Gamma(q)=\int_{0}^{\infty} e^{-s} s^{q-1} d s$.

Definition 2.2. The Riemann-Liouville fractional derivative of order $q>$ 0 of a continuous function $f:(0, \infty) \rightarrow \mathbb{R}$ is defined by

$$
D^{q} f(t)=\frac{1}{\Gamma(n-q)}\left(\frac{d}{d t}\right)^{n} \int_{0}^{t}(t-s)^{n-q-1} f(s) d s, \quad n-1<q<n,
$$

where $n=[q]+1,[q]$ denotes the integer part of a real number $q$, provided the right-hand side is point-wise defined on $(0, \infty)$.

Definition 2.3. The Caputo derivative of order $q$ for a function $f$ : $[0, \infty) \rightarrow \mathbb{R}$ can be written as

$$
{ }^{c} D^{q} f(t)=D^{q}\left(f(t)-\sum_{k=0}^{n-1} \frac{t^{k}}{k !} f^{(k)}(0)\right), \quad t>0, \quad n-1<q<n .
$$

Remark 2.4. If $f(t) \in C^{n}[0, \infty)$, then

$$
{ }^{c} D^{q} f(t)=\frac{1}{\Gamma(n-q)} \int_{0}^{t} \frac{f^{(n)}(s)}{(t-s)^{q+1-n}} d s=I^{n-q} f^{(n)}(t), t>0, n-1<q<n .
$$

Lemma 2.5. For $q>0$, the general solution of the fractional differential equation ${ }^{c} D^{q} x(t)=0$ is given by

$$
x(t)=c_{0}+c_{1} t+\ldots+c_{n-1} t^{n-1},
$$

where $c_{i} \in \mathbb{R}, i=1,2, \ldots, n-1(n=[q]+1)$. 
In view of Lemma 2.5 , it follows that

$$
I^{q c} D^{q} x(t)=x(t)+c_{0}+c_{1} t+\ldots+c_{n-1} t^{n-1},
$$

for some $c_{i} \in \mathbb{R}, i=1,2, \ldots, n-1(n=[q]+1)$.

Definition 2.6. [20] The generalized Riemann-Liouville fractional integral of order $q>0$ and $\rho>0$, of a function $f(t)$, for all $0<t<\infty$, is defined as

$$
{ }^{\rho} I^{q} f(t)=\frac{\rho^{1-q}}{\Gamma(q)} \int_{0}^{t} \frac{s^{\rho-1} f(s)}{\left(t^{\rho}-s^{\rho}\right)^{1-q}} d s
$$

provided the right-hand side is point-wise defined on $(0, \infty)$.

Remark 2.7. For $\rho=1$ in the above definition, we arrive at the standard Riemann-Liouville fractional integral, which is used to define both the RiemannLiouville and Caputo fractional derivatives, while, in the limit $\rho \rightarrow 0$, we have

$$
\lim _{\rho \rightarrow 0} \rho I^{q} f(t)=\frac{1}{\Gamma(q)} \int_{0}^{t}\left(\log \frac{t}{s}\right)^{q-1} \frac{f(s)}{s} d s,
$$

which is the famous Hadamard fractional integral. See [20].

Lemma 2.8. Let $q$ and $p$ be the positive constants. Then

$$
{ }^{\rho} I^{q} t^{p}=\frac{\Gamma\left(\frac{p+\rho}{\rho}\right)}{\Gamma\left(\frac{p+\rho q+\rho}{\rho}\right)} \frac{t^{p+\rho q}}{\rho^{q}} .
$$

Proof. From Definition 2.6, we have

$$
\begin{aligned}
{ }^{\rho} I^{q} t^{p} & =\frac{\rho^{1-q}}{\Gamma(q)} \int_{0}^{t} \frac{s^{\rho-1} s^{p}}{\left(t^{\rho}-s^{\rho}\right)^{1-q}} d s=\frac{\rho^{1-q}}{\Gamma(q)} \frac{t^{p+\rho q}}{\rho} \int_{0}^{1} \frac{u^{\frac{p}{\rho}}}{(1-u)^{1-q}} d u \\
& =\frac{\rho^{1-q}}{\Gamma(q)} \frac{t^{p+\rho q}}{\rho} B\left(\frac{p+\rho}{\rho}, q\right)=\frac{t^{p+\rho q}}{\rho^{q}} \frac{\Gamma\left(\frac{p+\rho}{\rho}\right)}{\Gamma\left(\frac{p+\rho q+\rho}{\rho}\right)} .
\end{aligned}
$$

This completes the proof.

Lemma 2.9. For any $y \in C([0, T], \mathbb{R})$, a function $x \in C^{2}([0, T], \mathbb{R})$ is a solution of the linear fractional boundary value problem

$$
\left\{\begin{array}{l}
{ }^{c} D^{\alpha} x(t)=y(t), \quad 1<\alpha \leq 2, \\
x(0)=0, \quad x(T)=\beta^{\rho} I^{q} x(\xi), \quad 0<\xi<T,
\end{array}\right.
$$


if and only if

$$
x(t)=J^{\alpha} y(t)+\frac{t}{\Lambda}\left\{\beta^{\rho} I^{q} J^{\alpha} y(\xi)-J^{\alpha} y(T)\right\}
$$

where

$$
\Lambda=T-\beta \frac{\xi^{\rho q+1}}{\rho^{q}} \frac{\Gamma\left(\frac{1+\rho}{\rho}\right)}{\Gamma\left(\frac{1+\rho q+\rho}{\rho}\right)} \neq 0 .
$$

Proof. It is well known that the general solution of the fractional differential equation in (5) can be written as

$$
x(t)=c_{0}+c_{1} t+J^{\alpha} y(t),
$$

where $c_{0}, c_{1} \in \mathbb{R}$ are arbitrary constants. Using the first condition $(x(0)=0)$ given by $(5)$ in $(8)$, we get $c_{0}=0$. Applying the generalized fractional integral operator on (8) and using Lemma 2.8, we obtain

$$
{ }^{\rho} I^{q} x(t)={ }^{\rho} I^{q} J^{\alpha} y(t)+c_{1} \frac{t^{\rho q+1}}{\rho^{q}} \frac{\Gamma\left(\frac{1+\rho}{\rho}\right)}{\Gamma\left(\frac{1+\rho q+\rho}{\rho}\right)},
$$

which, together with the second condition of (5), yields

$$
J^{\alpha} y(T)+c_{1} T=\beta^{\rho} I^{q} J^{\alpha} y(\xi)+\beta c_{1} \frac{\xi^{\rho q+1}}{\rho^{q}} \frac{\Gamma\left(\frac{1+\rho}{\rho}\right)}{\Gamma\left(\frac{1+\rho q+\rho}{\rho}\right)} .
$$

Using the notation (7) in (10), we find that

$$
c_{1}=\frac{1}{\Lambda}\left\{\beta^{\rho} I^{q} J^{\alpha} y(\xi)-J^{\alpha} y(T)\right\} .
$$

Substituting the values of $c_{0}$ and $c_{1}$ in (8), we obtain (6). Conversely, it can easily be shown by direct computation that $x$ given by the integral equation (6) satisfies the problem (5). This completes the proof.

Throughout this paper, for convenience of proving, we let the notations

$$
\begin{aligned}
& J^{z} f(s, x(s))(y)=\frac{1}{\Gamma(z)} \int_{0}^{y}(y-s)^{z-1} f(s, x(s)) d s \\
& { }^{\rho} I^{z} f(s, x(s))(y)=\frac{\rho^{1-z}}{\Gamma(z)} \int_{0}^{y} \frac{s^{\rho-1} f(s, x(s))}{\left(y^{\rho}-s^{\rho}\right)^{1-z}} d s
\end{aligned}
$$

where $z>0$ and $y \in[0, T]$. 


\section{Existence result for problem (1)}

We denote by $\mathcal{C}=C([0, T], \mathbb{R})$ the Banach space of all continuous functions from $[0, T] \rightarrow \mathbb{R}$ endowed with a topology of uniform convergence with the norm defined by $\|x\|=\sup \{|x(t)|: t \in[0, T]\}$. Also by $L^{1}([0, T], \mathbb{R})$ we denote the Banach space of measurable functions $x:[0, T] \rightarrow \mathbb{R}$ which are Lebesgue integrable and normed by $\|x\|_{L^{1}}=\int_{0}^{T}|x(t)| d t$.

Our main result in this section is based upon the following fixed point theorem due to Burton and Kirk [24].

Theorem 3.1. Let $X$ be a Banach space, and $A, B: X \rightarrow X$ two operators such that:

(i) $A$ is a contraction, and

(ii) $B$ is completely continuous.

Then either

(a) the operator equation $y=A(y)+B(y)$ has a solution, or

(b) the set $\mathcal{E}=\left\{u \in X: \lambda A\left(\frac{u}{\lambda}\right)+\lambda B(u)=u\right\}$ is unbounded for $\lambda \in(0,1)$.

Theorem 3.2. Assume that $f, g:[0, T] \times \mathbb{R} \rightarrow \mathbb{R}$ are continuous functions. In addition we suppose that:

$\left(H_{1}\right)$ there exist a function $k(t) \in L^{1}\left(J, \mathbb{R}_{+}\right)$such that

$$
|g(t, u)-g(t, \bar{u})| \leq k(t)|u-\bar{u}|, \quad \text { for a.e. } t \in J, u, \bar{u} \in \mathbb{R},
$$

with

$$
\gamma:=J^{\alpha} k(s)(T)+\frac{T}{|\Lambda|} J^{\alpha} k(s)(T)+\frac{|\beta| T}{|\Lambda|} \rho I^{q} J^{\alpha} k(s)(\xi)<\frac{1}{2} ;
$$

$\left(H_{2}\right)$ there exists a function $p \in L^{1}\left(J, \mathbb{R}_{+}\right)$such that

$$
|f(t, u)| \leq p(t), \text { for a.e. } t \in J, \text { and each } u \in \mathbb{R} .
$$

Then the boundary value problem (1) has at least one solution on $[0, T]$. 
Proof. Let us transform the problem (1) into a fixed point problem. For that, we introduce an operator $\mathcal{P}: \mathcal{C} \rightarrow \mathcal{C}$ associated with the problem (1) as

$$
\begin{aligned}
(\mathcal{P} x)(t) & =J^{\alpha}[f(s, x(s))+g(s, x(s))](t) \\
& +\frac{t}{\Lambda}\left\{\beta^{\rho} I^{q} J^{\alpha}[f(s, x(s))+g(s, x(s))](\xi)\right. \\
& \left.-J^{\alpha}[f(s, x(s))+g(s, x(s))](T)\right\}, \quad t \in[0, T] .
\end{aligned}
$$

Then we split the operator $\mathcal{P}: \mathcal{C} \rightarrow \mathcal{C}$ defined by (12) as

$$
(\mathcal{P} x)(t)=\left(\mathcal{P}_{1} x\right)(t)+\left(\mathcal{P}_{2} x\right)(t), \quad t \in[0, T]
$$

where $\mathcal{P}_{1,2}: \mathcal{C} \rightarrow \mathcal{C}$ are given by

$$
\begin{aligned}
& \left(\mathcal{P}_{1} x\right)(t)=J^{\alpha} f(s, x(s))(t)+\frac{t}{\Lambda}\left\{\beta^{\rho} I^{q} J^{\alpha} f(s, x(s))(\xi)-J^{\alpha} f(s, x(s))(T)\right\}, \\
& \left(\mathcal{P}_{2} x\right)(t)=J^{\alpha} g(s, x(s))(t)+\frac{t}{\Lambda}\left\{\beta^{\rho} I^{q} J^{\alpha} g(s, x(s))(\xi)-J^{\alpha} g(s, x(s))(T)\right\} .
\end{aligned}
$$

Obviously the existence of a fixed point for the operator $\mathcal{P}$ is equivalent to the existence of one for $\mathcal{P}_{1}+\mathcal{P}_{2}$. So we shall show that the operators $\mathcal{P}_{1}$ and $\mathcal{P}_{2}$ satisfy all conditions of Theorem 3.1. The proof will be given in several steps.

Step 1. The operator $\mathcal{P}_{1}$ defined by (14) is continuous.

Let $\left\{x_{n}\right\} \subset B_{r}=\{x \in \mathcal{C}:\|x\| \leq r\}$ with $\left\|x_{n}-x\right\| \rightarrow 0$. Then the limit $\left\|x_{n}(t)-x(t)\right\| \rightarrow 0$ is uniformly valid on $[0, T]$. From the uniform continuity of $f(t, x)$ on the compact set $[0, T] \times[-r, r]$, it follows that $\| f\left(t, x_{n}(t)\right)-$ $f(t, x(t)) \| \rightarrow 0$ is uniformly valid on $[0, T]$. Hence $\left\|\mathcal{P}_{1} x_{n}-\mathcal{P}_{1} x\right\| \rightarrow 0$ as $n \rightarrow \infty$, which proves the continuity of $\mathcal{P}_{1}$.

Step 2. The operator $\mathcal{P}_{1}$ maps bounded sets into bounded sets in $\mathcal{C}$.

It is enough to show that for any $r>0$, there exists a positive constant $L$ such that for each $x \in B_{r}$, we have $\left\|\mathcal{P}_{1} x\right\| \leq L$. Let $x \in B_{r}$. Then

$$
\begin{aligned}
\left\|\mathcal{P}_{1} x\right\| & \leq J^{\alpha}|f(s, x(s))|(T)+\frac{T}{|\Lambda|}\left\{|\beta|^{\rho} I^{q} J^{\alpha}|f(s, x(s))|(\xi)+J^{\alpha}|f(s, x(s))|(T)\right\} \\
& \leq J^{\alpha} p(s)(T)+\frac{|\beta| T}{|\Lambda|}{ }^{\rho} I^{q} J^{\alpha} p(s)(\xi)+\frac{T}{|\Lambda|} J^{\alpha} p(s)(T):=L .
\end{aligned}
$$

Step 3. The operator $\mathcal{P}_{1}$ maps bounded sets into equicontinuous sets in $\mathcal{C}$. 
Let $\tau_{1}, \tau_{2} \in[0, T]$ with $\tau_{1}<\tau_{2}$ and $x \in B_{r}$. Then, for each $x \in B_{r}$, we obtain

$$
\begin{aligned}
& \left|\left(\mathcal{P}_{1} x\right)\left(\tau_{2}\right)-\left(\mathcal{P}_{1} x\right)\left(\tau_{1}\right)\right| \\
\leq \quad & \left|J^{\alpha} f(s, x(s))\left(\tau_{2}\right)-J^{\alpha} f(s, x(s))\left(\tau_{1}\right)\right|+\frac{\left|\tau_{2}-\tau_{1}\right|}{|\Lambda|} J^{\alpha}|f(s, x(s))|(T) \\
& +\left.\frac{|\beta|\left|\tau_{2}-\tau_{1}\right|}{|\Lambda|}\right|^{\rho} I^{q} J^{\alpha}|f(s, x(s))|(\xi) \\
\leq & \frac{1}{\Gamma(\alpha)}\left|\int_{0}^{\tau_{1}}\left[\left(\tau_{2}-s\right)^{\alpha-1}-\left(\tau_{1}-s\right)^{\alpha-1}\right] p(s) d s+\int_{\tau_{1}}^{\tau_{2}}\left(\tau_{2}-s\right)^{\alpha-1} p(s) d s\right| \\
& +\frac{\left|\tau_{2}-\tau_{1}\right|}{|\Lambda|}\left(J^{\alpha} p(s)(T)+|\beta|^{\rho} I^{q} J^{\alpha} p(s)(\xi)\right),
\end{aligned}
$$

which is independent of $x$ and tends to zero as $\tau_{2}-\tau_{1} \rightarrow 0$. Thus, $\mathcal{P}_{1}$ is equicontinuous. Thus, by the above three steps, the operator $\mathcal{P}_{1}$ is completely continuous.

Step 4. The operator $\mathcal{P}_{2}$ defined by (15) is a contraction.

For $x, y \in \mathcal{C}$ and for each $t \in[0, T]$, from the definition of $\mathcal{P}_{2}$ and assumption $\left(H_{1}\right)$, we obtain

$$
\begin{aligned}
& \left|\left(\mathcal{P}_{2} x\right)(t)-\left(\mathcal{P}_{2} y\right)(t)\right| \\
\leq & J^{\alpha}|g(s, x(s))-g(s, y(s))|(t)+\frac{T}{|\Lambda|} J^{\alpha}|g(s, x(s))-g(s, y(s))|(T) \\
& +\frac{|\beta| T}{|\Lambda|}{ }^{\rho} I^{q} J^{\alpha}|g(s, x(s))-g(s, y(s))|(\xi) \\
\leq & \left\{J^{\alpha} k(s)(T)+\frac{T}{|\Lambda|} J^{\alpha} k(s)(T)+\frac{|\beta| T}{|\Lambda|}{ }^{\rho} I^{q} J^{\alpha} k(s)(\xi)\right\}\|x-y\| .
\end{aligned}
$$

Hence

$$
\left\|\left(\mathcal{P}_{2} x\right)-\left(\mathcal{P}_{2} y\right)\right\| \leq \gamma\|x-y\|
$$

As $\gamma<1$ by (11), the operator $\mathcal{P}_{2}$ is a contraction map from the Banach space $\mathcal{C}$ into itself.

Step 5. A priori bounds on solutions.

Now it remains to show that the set $\mathcal{E}=\left\{u \in \mathcal{C}: \lambda \mathcal{P}_{1}\left(\frac{u}{\lambda}\right)+\lambda \mathcal{P}_{2}(u)=u\right\}$ is unbounded for some $\lambda \in(0,1)$. 
Let $\lambda \in(0,1)$ and $x \in \mathcal{E}$ be a solution of the integral equation

$$
\begin{array}{ll} 
& x(t) \\
= & \lambda J^{\alpha} f(s, x(s))(t)+\lambda \frac{t}{\Lambda}\left\{\beta^{\rho} I^{q} J^{\alpha} f(s, x(s))(\xi)-J^{\alpha} f(s, x(s))(T)\right\} \\
& +\lambda J^{\alpha} g\left(s, \frac{x(s)}{\lambda}\right)(t)+\lambda \frac{t}{\Lambda}\left\{\beta^{\rho} I^{q} J^{\alpha} g\left(s, \frac{x(s)}{\lambda}\right)(\xi)-J^{\alpha} g\left(s, \frac{x(s)}{\lambda}\right)(T)\right\}, \\
& t \in[0, T] .
\end{array}
$$

Then, for each $t \in[0, T]$, we have

$$
\begin{aligned}
|x(t)| \leq & J^{\alpha} p(s)(T)+\frac{|\beta| T}{|\Lambda|}{ }^{\rho} I^{q} J^{\alpha} p(s)(\xi)+\frac{T}{|\Lambda|} J^{\alpha} p(s)(T) \\
& +\lambda J^{\alpha}\left[\left|g\left(s, \frac{x(s)}{\lambda}\right)-g(s, 0)\right|+|g(s, 0)|\right](T) \\
& +\frac{|\beta| T}{|\Lambda|}{ }^{\rho} I^{q} J^{\alpha}\left[\left|g\left(s, \frac{x(s)}{\lambda}\right)-g(s, 0)\right|+|g(s, 0)|\right](\xi) \\
& +\frac{T}{|\Lambda|} J^{\alpha}\left[\left|g\left(s, \frac{x(s)}{\lambda}\right)-g(s, 0)\right|+|g(s, 0)|\right](T) \\
\leq & J^{\alpha} p(s)(T)+\frac{|\beta| T}{|\Lambda|}{ }^{\rho} I^{q} J^{\alpha} p(s)(\xi)+\frac{T}{|\Lambda|} J^{\alpha} p(s)(T) \\
& +\|x\|\left\{J^{\alpha} k(s)(T)+\frac{|\beta| T}{|\Lambda|}{ }^{\rho} I^{q} J^{\alpha} k(s)(\xi)+\frac{T}{|\Lambda|} J^{\alpha} k(s)(T)\right\} \\
& +g^{*}\left\{\frac{T^{\alpha}}{\Gamma(\alpha+1)}+\frac{T}{|\Lambda|} \frac{T^{\alpha}}{\Gamma(\alpha+1)}+\frac{T}{|\Lambda|} \frac{|\beta|}{\Gamma(\alpha+1)} \frac{\xi^{\alpha+\rho q}}{\rho^{q}} \frac{\Gamma\left(\frac{\alpha+\rho}{\rho}\right)}{\Gamma\left(\frac{\alpha+\rho q+\rho}{\rho}\right)}\right\},
\end{aligned}
$$

where $g^{*}=\sup \{|g(t, 0)|, t \in[0, T]\}$, which yields

$$
\begin{aligned}
(1-\gamma)\|x\| & \leq J^{\alpha} p(s)(T)+\frac{|\beta| T}{|\Lambda|}{ }^{\rho} I^{q} J^{\alpha} p(s)(\xi)+\frac{T}{|\Lambda|} J^{\alpha} p(s)(T) \\
& +g^{*}\left\{\frac{T^{\alpha}}{\Gamma(\alpha+1)}+\frac{T}{|\Lambda|} \frac{T^{\alpha}}{\Gamma(\alpha+1)}+\frac{T}{|\Lambda|} \frac{|\beta|}{\Gamma(\alpha+1)} \frac{\xi^{\alpha+\rho q}}{\rho^{q}} \frac{\Gamma\left(\frac{\alpha+\rho}{\rho}\right)}{\Gamma\left(\frac{\alpha+\rho q+\rho}{\rho}\right)}\right\} .
\end{aligned}
$$

Consequently we have

$$
\|x\| \leq M:=\frac{1}{(1-\gamma)}\left[J^{\alpha} p(s)(T)+\frac{|\beta| T}{|\Lambda|}{ }^{\rho} I^{q} J^{\alpha} p(s)(\xi)+\frac{T}{|\Lambda|} J^{\alpha} p(s)(T)\right.
$$




$$
\left.+g^{*}\left\{\frac{T^{\alpha}}{\Gamma(\alpha+1)}+\frac{T}{|\Lambda|} \frac{T^{\alpha}}{\Gamma(\alpha+1)}+\frac{T}{|\Lambda|} \frac{|\beta|}{\Gamma(\alpha+1)} \frac{\xi^{\alpha+\rho q}}{\rho^{q}} \frac{\Gamma\left(\frac{\alpha+\rho}{\rho}\right)}{\Gamma\left(\frac{\alpha+\rho q+\rho}{\rho}\right)}\right\}\right],
$$

which means that the set $\mathcal{E}$ is bounded, since $\gamma<1 / 2$. Hence, part (b) of Theorem 3.1 does not hold. Thus part (a) holds, that is, $\mathcal{P}$ has a fixed point in $[0, T]$, and consequently the problem (1) has a solution. This completes the proof.

\section{Existence result for problem (2)}

First of all, we recall some basic concepts for multi-valued maps [26, 27, 28].

For a normed space $(X,\|\cdot\|)$, let $\mathcal{P}_{c l}(X)=\{Y \in \mathcal{P}(X): Y$ is closed $\}$, $\mathcal{P}_{b}(X)=\{Y \in \mathcal{P}(X): Y$ is bounded $\}, \mathcal{P}_{c p}(X)=\{Y \in \mathcal{P}(X): Y$ is compact $\}$ and $\mathcal{P}_{c p, c}(X)=\{Y \in \mathcal{P}(X): Y$ is compact and convex $\}$.

A multi-valued map $G: X \rightarrow \mathcal{P}(X):$

(i) is convex (closed) valued if $G(x)$ is convex (closed) for all $x \in X$;

(ii) is bounded on bounded sets if $G(\mathbb{B})=\cup_{x \in \mathbb{B}} G(x)$ is bounded in $X$ for all $\mathbb{B} \in \mathcal{P}_{b}(X)$ (i.e. $\sup _{x \in \mathbb{B}}\{\sup \{|y|: y \in G(x)\}\}<\infty$ );

(iii) is called upper semi-continuous (u.s.c.) on $X$ if for each $x_{0} \in X$, the set $G\left(x_{0}\right)$ is a nonempty closed subset of $X$, and if for each open set $N$ of $X$ containing $G\left(x_{0}\right)$, there exists an open neighborhood $\mathcal{N}_{0}$ of $x_{0}$ such that $G\left(\mathcal{N}_{0}\right) \subseteq N$

(iv) $G$ is lower semi-continuous (l.s.c.) if the set $\{y \in X: G(y) \cap B \neq \emptyset\}$ is open for any open set $B$ in $E$;

(v) is said to be completely continuous if $G(\mathbb{B})$ is relatively compact for every $\mathbb{B} \in \mathcal{P}_{b}(X)$

(vi) is said to be measurable if for every $y \in \mathbb{R}$, the function

$$
t \longmapsto d(y, G(t))=\inf \{|y-z|: z \in G(t)\}
$$

is measurable;

(vii) has a fixed point if there is $x \in X$ such that $x \in G(x)$. The fixed point set of the multi-valued operator $G$ will be denoted by Fix $G$. 
Definition 4.1. A multivalued map $F:[0, T] \times \mathbb{R} \rightarrow \mathcal{P}(\mathbb{R})$ is said to be Carathéodory if

(i) $t \longmapsto F(t, x)$ is measurable for each $x \in \mathbb{R}$;

(ii) $x \longmapsto F(t, x)$ is upper semicontinuous for almost all $t \in[0, T]$;

Further a Carathéodory function $F$ is called $L^{1}$-Carathéodory if

(iii) for each $\alpha>0$, there exists $\varphi_{\alpha} \in L^{1}\left([0, T], \mathbb{R}^{+}\right)$such that

$$
\|F(t, x)\|=\sup \{|v|: v \in F(t, x)\} \leq \varphi_{\alpha}(t)
$$

for all $\|x\| \leq \alpha$ and for a. e. $t \in[0, T]$.

For each $x \in \mathcal{C}$, define the set of selections of $F$ by

$$
S_{F, x}:=\left\{v \in L^{1}([0, T], \mathbb{R}): v(t) \in F(t, x(t)) \text { for a.e. } t \in[0, T]\right\} .
$$

We define the graph of $G$ to be the set $G r(G)=\{(x, y) \in X \times Y, y \in G(x)\}$ and recall two useful results regarding closed graphs and upper-semicontinuity.

Lemma 4.2. ([26, Proposition 1.2]) If $G: X \rightarrow \mathcal{P}_{c l}(Y)$ is u.s.c., then $\operatorname{Gr}(G)$ is a closed subset of $X \times Y$; i.e., for every sequence $\left\{x_{n}\right\}_{n \in \mathbb{N}} \subset X$ and $\left\{y_{n}\right\}_{n \in \mathbb{N}} \subset Y$, if when $n \rightarrow \infty, x_{n} \rightarrow x_{*}, y_{n} \rightarrow y_{*}$ and $y_{n} \in G\left(x_{n}\right)$, then $y_{*} \in G\left(x_{*}\right)$. Conversely, if $G$ is completely continuous and has a closed graph, then it is upper semi-continuous.

Lemma 4.3. ([29]) Let $X$ be a Banach space. Let $F:[0, T] \times \mathbb{R} \rightarrow \mathcal{P}_{c p, c}(X)$ be an $L^{1}$ - Carathéodory multivalued map and let $\Theta$ be a linear continuous mapping from $L^{1}([0, T], X)$ to $C([0, T], X)$. Then the operator

$$
\Theta \circ S_{F, x}: C([0, T], X) \rightarrow \mathcal{P}_{c p, c}(C([0, T], X)), \quad x \mapsto\left(\Theta \circ S_{F, x}\right)(x)=\Theta\left(S_{F, x}\right)
$$

is a closed graph operator in $C([0, T], X) \times C([0, T], X)$.

To prove our main result in this section, we use the following form of the nonlinear alternative for contractive maps [25, Corollary 3.8].

Theorem 4.4. Let $X$ be a Banach space, and $D$ a bounded neighborhood of $0 \in X$. Let $Z_{1}: X \rightarrow \mathcal{P}_{c p, c}(X)$ and $Z_{2}: \bar{D} \rightarrow \mathcal{P}_{c p, c}(X)$ two multi-valued operators such that

(a) $Z_{1}$ is contraction, and

(b) $Z_{2}$ is upper semi-continuous and compact. 
Then, if $Q=Z_{1}+Z_{2}$, either

(i) $Q$ has a fixed point in $\bar{D}$ or

(ii) there is a point $u \in \partial D$ and $\lambda \in(0,1)$ with $u \in \lambda Q(u)$.

Definition 4.5. A function $x \in C([0, T], \mathbb{R})$ possessing a fractional derivative of order $\alpha \in(1,2]$ is a solution of the problem $(2)$ if $x(0)=0, x(T)=$ $\beta^{\rho} I^{q} x(\xi)$, and there exist functions $f, g \in L^{1}([0, T], \mathbb{R})$ such that $f(t) \in$ $F(t, x(t))$ and $g(t) \in G(t, x(t))$ a.e. on $[0, T]$ and

$$
\begin{aligned}
x(t)=J^{\alpha}[f(s)+ & g(s)](t) \\
& +\frac{t}{\Lambda}\left\{\beta^{\rho} I^{q} J^{\alpha}[f(s)+g(s)](\xi)-J^{\alpha}[f(s)+g(s)](T)\right\} .
\end{aligned}
$$

Theorem 4.6. Assume that:

$\left(A_{1}\right) F:[0, T] \times \mathbb{R} \rightarrow \mathcal{P}_{c p, c}(\mathbb{R})$ is $L^{1}$-Carathéodory;

$\left(A_{2}\right)$ there exists a continuous nondecreasing function $\Phi:[0, \infty) \rightarrow(0, \infty)$ and a function $p \in L^{1}\left([0, T], \mathbb{R}^{+}\right)$such that

$$
\begin{array}{r}
\|F(t, x)\|_{\mathcal{P}}:=\sup \{|y|: y \in F(t, x)\} \leq p(t) \Phi(\|x\|) \\
\text { for each }(t, x) \in[0, T] \times \mathbb{R} ;
\end{array}
$$

$\left(A_{3}\right)$ the multi-valued map $t \rightarrow G(t, x)$ is measurable for each $x \in \mathbb{R}$ and integrably bounded, i.e., there exists a function $M \in L^{1}([0, T], \mathbb{R})$ such that

$|G(t, x)|:=\sup \{|g|: g(t) \in G(t, x)\} \leq M(t)$, for a.e. $t \in[0, T]$ and $x \in \mathbb{R}$

$\left(A_{4}\right)$ for $G:[0, T] \times \mathbb{R} \rightarrow \mathcal{P}_{c p, c}(\mathbb{R})$, there exists a function $\ell \in L^{1}([0, T], \mathbb{R})$ such that

$$
H_{d}(G(t, x)-G(t, y)) \leq \ell(t)|x-y|, t \in[0, T]
$$

for all $x, y \in \mathbb{R}$ with

$$
J^{q} \ell(s)(T)+\frac{T}{|\Lambda|}\left(|\beta|^{\rho} I^{q} J^{q} \ell(s)\left(\xi_{i}\right)+J^{q} \ell(s)(T)(T)\right)<1 ;
$$


$\left(A_{5}\right)$ there exists a constant $M>0$ such that

$$
\frac{M}{\Phi(M) \Psi_{1}+\Psi_{2}}>1
$$

where

$$
\Psi_{1}=J^{\alpha} p(s)(T)+\frac{|\beta| T}{|\Lambda|} \rho^{\rho} I^{q} J^{\alpha} p(s)(\xi)+\frac{T}{|\Lambda|} J^{\alpha} p(s)(T)
$$

and

$$
\Psi_{2}=J^{\alpha} M(s)(T)+\frac{|\beta| T}{|\Lambda|} \rho^{\rho} I^{q} J^{\alpha} M(s)(\xi)+\frac{T}{|\Lambda|} J^{\alpha} M(s)(T) .
$$

Then the boundary value problem (2) has at least one solution on $[0, T]$.

Proof. To transform the problem (2) into a fixed point problem, we define an operator $\mathcal{N}: \mathcal{C} \longrightarrow \mathcal{P}(\mathcal{C})$ by

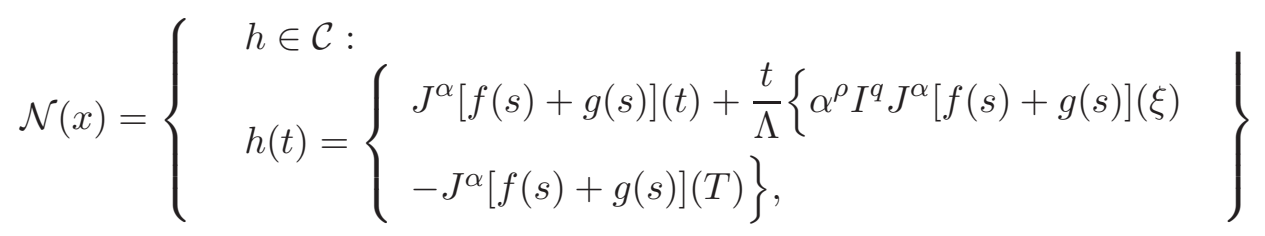

for $f \in S_{F, x}$ and $g \in S_{G, x}$.

Next we introduce the multi-valued operators

$$
\mathcal{A}, \mathcal{B}: C([0, T], \mathbb{R}) \longrightarrow \mathcal{P}(C([0, T], \mathbb{R}))
$$

by

$$
\mathcal{A}(x)=\left\{h \in \mathcal{C}: h(t)=J^{\alpha} f(s)(t)+\frac{t}{\Lambda}\left\{\beta^{\rho} I^{q} J^{\alpha} f(s)(\xi)-J^{\alpha} f(s)(T)\right\}\right\}
$$

for $f \in S_{F, x}$ and

$$
\mathcal{B}(x)=\left\{z \in \mathcal{C}: z(t)=J^{\alpha} g(s)(t)+\frac{t}{\Lambda}\left\{\beta^{\rho} I^{q} J^{\alpha} g(s)(\xi)-J^{\alpha} g(s)(T)\right\}\right\}
$$

for $g \in S_{G, x}$. Observe that $\mathcal{N}=\mathcal{A}+\mathcal{B}$. We shall show that the operators $\mathcal{A}$ and $\mathcal{B}$ satisfy all the conditions of Theorem 4.4 on $[0, T]$. First, we show that the operators $\mathcal{A}$ and $\mathcal{B}$ define the multivalued operators, that is, $\mathcal{A}, \mathcal{B}: B_{r} \rightarrow$ $\mathcal{P}_{c p, c}(\mathcal{C})$, where $B_{r}=\{x \in \mathcal{C}:\|x\| \leq r\}$ is a bounded set in $\mathcal{C}$. First we prove 
that $\mathcal{A}$ is compact-valued on $B_{r}$. Note that the operator $\mathcal{A}$ is equivalent to the composition $\mathcal{L} \circ S_{F}$, where $\mathcal{L}$ is the continuous linear operator on $L^{1}([0, T], \mathbb{R})$ into $\mathcal{C}$, defined by

$$
\mathcal{L}(v)(t)=J^{\alpha} v(s)(t)+\frac{t}{\Lambda}\left\{\beta^{\rho} I^{q} J^{\alpha} v(s)(\xi)-J^{\alpha} v(s)(T)\right\} .
$$

Suppose that $x \in B_{r}$ is arbitrary and let $\left\{v_{n}\right\}$ be a sequence in $S_{F, x}$. Then, by definition of $S_{F, x}$, we have $v_{n}(t) \in F(t, x(t))$ for almost all $t \in[0, T]$. Since $F(t, x(t))$ is compact for all $t \in J$, there is a convergent subsequence of $\left\{v_{n}(t)\right\}$ (we denote it by $\left\{v_{n}(t)\right\}$ again) that converges in measure to some $v(t) \in S_{F, x}$ for almost all $t \in J$. On the other hand, $\mathcal{L}$ is continuous, so $\mathcal{L}\left(v_{n}\right)(t) \rightarrow \mathcal{L}(v)(t)$ pointwise on $[0, T]$.

In order to show that the convergence is uniform, we have to show that $\left\{\mathcal{L}\left(v_{n}\right)\right\}$ is an equi-continuous sequence. Let $t_{1}, t_{2} \in[0, T]$ with $t_{1}<t_{2}$. Then, we have

$$
\begin{aligned}
& \left|\mathcal{L}\left(v_{n}\right)\left(t_{2}\right)-\mathcal{L}\left(v_{n}\right)\left(t_{1}\right)\right| \\
\leq & \left|J^{\alpha} v_{n}(s)\left(t_{2}\right)-J^{\alpha} v_{n}(s)\left(t_{1}\right)\right|+\frac{\left|t_{2}-t_{1}\right|}{|\Lambda|} J^{q}\left|v_{n}(s)\right|(T) \\
& +\left.\frac{|\beta|\left|t_{2}-t_{1}\right|}{|\Lambda|}\right|^{\rho} I^{q} J^{q}\left|v_{n}(s)\right|(\xi) \\
\leq & \frac{\psi(r)}{\Gamma(q)}\left|\int_{0}^{t_{1}}\left[\left(t_{2}-s\right)^{q-1}-\left(t_{1}-s\right)^{q-1}\right] p(s) d s+\int_{t_{1}}^{t_{2}}\left(t_{2}-s\right)^{q-1} p(s) d s\right| \\
& +\psi(r) \frac{\left|t_{2}-t_{1}\right|}{|\Lambda|}\left(J^{q} p(s)(T)+\left.|\beta|\right|^{\rho} I^{q} J^{q} p(s)(\xi)\right) .
\end{aligned}
$$

We see that the right hand of the above inequality tends to zero, independently of $x$, as $t_{2} \rightarrow t_{1}$. Thus, the sequence $\left\{\mathcal{L}\left(v_{n}\right)\right\}$ is equi-continuous and by using the Arzelá-Ascoli theorem, we get that there is a uniformly convergent subsequence. So, there is a subsequence of $\left\{v_{n}\right\}$ (we denote it again by $\left\{v_{n}\right\}$ ) such that $\mathcal{L}\left(v_{n}\right) \rightarrow \mathcal{L}(v)$. Note that, $\mathcal{L}(v) \in \mathcal{L}\left(S_{F, x}\right)$. Hence, $\mathcal{A}(x)=\mathcal{L}\left(S_{F, x}\right)$ is compact for all $x \in B_{r}$. So $\mathcal{A}(x)$ is compact.

Now, we show that $\mathcal{A}(x)$ is convex for all $x \in \mathcal{C}$. Let $h_{1}, h_{2} \in \mathcal{A}(x)$. We select $f_{1}, f_{2} \in S_{F, x}$ such that

$$
h_{i}(t)=J^{\alpha} f_{i}(s)(t)+\frac{t}{\Lambda}\left\{\beta^{\rho} I^{q} J^{\alpha} f_{i}(s)(\xi)-J^{\alpha} f_{i}(s)(T)\right\}, \quad i=1,2,
$$

for almost all $t \in[0, T]$. Let $0 \leq \lambda \leq 1$. Then, we have

$$
\left[\lambda h_{1}+(1-\lambda) h_{2}\right](t)=J^{\alpha}\left[\lambda f_{1}(s)+(1-\lambda) f_{2}(s)\right](t)
$$


ON PERTURBED FRACTIONAL DIFFERENTIAL EQUATIONS...

$$
+\frac{t}{\Lambda}\left\{\beta^{\rho} I^{q} J^{\alpha}\left[\lambda f_{1}(s)+(1-\lambda) f_{2}(s)\right](\xi)-J^{\alpha}\left[\lambda f_{1}(s)+(1-\lambda) f_{2}(s)\right](s)(T)\right\} .
$$

Since $F$ has convex values, so $S_{F, x}$ is convex and $\lambda f_{1}(s)+(1-\lambda) f_{2}(s) \in S_{F, x}$. Thus

$$
\lambda h_{1}+(1-\lambda) h_{2} \in \mathcal{A}(x) .
$$

Consequently, $\mathcal{A}$ is convex-valued. For $\mathcal{B}$ we work in a similar way.

The rest of the proof consists of several steps and claims.

Step 1: We show that $\mathcal{B}$ is a contraction on $\mathcal{C}$. Let $x, \bar{x} \in C([0, T], \mathbb{R})$ and $z_{1} \in \mathcal{G}(x)$. Then there exists $g_{1}(t) \in G(t, x(t))$ such that, for each $t \in[0, T]$,

$$
z_{1}(t)=J^{q} g_{1}(s)(t)+\frac{t^{q-1}}{\Lambda}\left(\beta^{\rho} I^{q} J^{\alpha} g_{1}(s)\left(\xi_{i}\right)-J^{q} g_{1}(s)(T)\right) .
$$

By $\left(A_{4}\right)$, we have

$$
H_{d}(G(t, x), G(t, \bar{x})) \leq \ell(t)|x(t)-\bar{x}(t)|
$$

So, there exists $w \in G(t, \bar{x}(t))$ such that

$$
\left|v_{1}(t)-w\right| \leq \ell(t)|x(t)-\bar{x}(t)|, \quad t \in[0, T] .
$$

Define $U:[0, T] \rightarrow \mathcal{P}(\mathbb{R})$ by

$$
U(t)=\left\{w \in \mathbb{R}:\left|v_{1}(t)-w\right| \leq \ell(t)|x(t)-\bar{x}(t)|\right\} .
$$

Since the multivalued operator $U(t) \cap G(t, \bar{x}(t))$ is measurable (Proposition III.4 [30]), there exists a function $v_{2}(t)$ which is a measurable selection for $U$. So $v_{2}(t) \in G(t, \bar{x}(t))$ and for each $t \in[0, T]$, we have $\left|v_{1}(t)-v_{2}(t)\right| \leq \ell(t)|x(t)-\bar{x}(t)|$.

For each $t \in[0, T]$, let us define

$$
z_{2}(t)=J^{q} g_{2}(s)(t)+\frac{t}{\Lambda}\left(\beta^{\rho} I^{q} J^{\alpha} g_{2}(s)\left(\xi_{i}\right)-J^{q} g_{2}(s)(T)\right) .
$$

Thus,

$$
\begin{aligned}
& \left|z_{1}(t)-z_{2}(t)\right| \\
\leq \quad & J^{q}\left|g_{1}(s)-g_{2}(s)\right|(t) \\
& +\frac{t}{|\Lambda|}\left(\beta^{\rho} I^{q} J^{\alpha}\left|g_{1}(s)-g_{2}(s)\right|\left(\xi_{i}\right)+J^{q}\left|g_{1}(s)-g_{2}(s)\right|(T)\right)
\end{aligned}
$$




$$
\leq\left\{J^{q} \ell(s)(T)+\frac{T}{|\Lambda|}\left(|\beta|^{\rho} I^{q} J^{q} \ell(s)\left(\xi_{i}\right)+J^{q} \ell(s)(T)(T)\right)\right\}\|x-\bar{x}\| .
$$

Hence,

$$
\left\|z_{1}-z_{2}\right\| \leq\left\{J^{q} \ell(s)(T)+\frac{T}{|\Lambda|}\left(|\beta|^{\rho} I^{q} J^{q} \ell(s)\left(\xi_{i}\right)+J^{q} \ell(s)(T)(T)\right)\right\}\|x-\bar{x}\| .
$$

Analogously, interchanging the roles of $x$ and $\bar{x}$, we obtain

$$
H_{d}(\mathcal{A}(x), \mathcal{A}(\bar{x})) \leq \delta\|x-\bar{x}\|
$$

where

$$
\delta=J^{q} \ell(s)(T)+\frac{T}{|\Lambda|}\left(|\beta|^{\rho} I^{q} J^{q} \ell(s)\left(\xi_{i}\right)+J^{q} \ell(s)(T)(T)\right) .
$$

So $\mathcal{A}$ is a contraction, since $\delta<1$ by (17).

Step 2: $\mathcal{A}$ is compact and upper semi-continuous. This will be established in several claims.

Claim I: $\mathcal{A}$ maps bounded sets into bounded sets in $\mathcal{C}$. Let $B_{r}=\{x \in \mathcal{C}$ : $\|x\| \leq r\}$ be a bounded set in $\mathcal{C}$. Then, for each $h \in \mathcal{A}(x), x \in B_{r}$, there exists $f \in S_{F, x}$ such that

$$
h(t)=J^{\alpha} f(s)(t)+\frac{t}{\Lambda}\left\{\beta^{\rho} I^{q} J^{\alpha} f(s)(\xi)-J^{\alpha} f(s)(T)\right\} .
$$

Then, for $t \in[0, T]$, we have

$$
\begin{aligned}
|h(t)| & \leq J^{\alpha}|f(s)|(T)+\frac{T}{|\Lambda|}\left\{|\beta|^{\rho} I^{q} J^{\alpha}|f(s)|(\xi)-J^{\alpha}|f(s)|(T)\right\} \\
& \leq \psi(\|x\|) J^{\alpha} p(s)(T)+\psi(\|x\|) \frac{|\beta| T^{\rho}}{|\Lambda|} I^{q} J^{\alpha} p(s)(\xi)+\psi(\|x\|) \frac{T}{|\Lambda|} J^{\alpha} p(s)(T) \\
& \leq \psi(\|x\|)\left\{J^{\alpha} p(s)(T)+\frac{|\beta| T}{|\Lambda|}{ }^{\rho} I^{q} J^{\alpha} p(s)(\xi)+\frac{T}{|\Lambda|} J^{\alpha} p(s)(T)\right\} .
\end{aligned}
$$

Thus,

$$
\|h\| \leq \psi(r)\left\{J^{\alpha} p(s)(T)+\frac{|\beta| T}{|\Lambda|} \rho I^{q} J^{\alpha} p(s)(\xi)+\frac{T}{|\Lambda|} J^{\alpha} p(s)(T)\right\} .
$$


Claim II: $\mathcal{B}$ maps bounded sets into equi-continuous sets. Let $\tau_{1}, \tau_{2} \in[0, T]$ with $\tau_{1}<\tau_{2}$ and $x \in B_{r}$. Then, for each $h \in \mathcal{A}(x)$, we obtain

$$
\begin{aligned}
& \left|h\left(\tau_{2}\right)-h\left(\tau_{1}\right)\right| \\
\leq & \left|J^{\alpha} f(s)\left(\tau_{2}\right)-J^{\alpha} f(s)\left(\tau_{1}\right)\right|+\frac{\left|\tau_{2}-\tau_{1}\right|}{|\Lambda|} J^{\alpha}|f(s)|(T) \\
& +\left.\frac{|\beta|\left|\tau_{2}-\tau_{1}\right|}{|\Lambda|}\right|^{\rho} I^{q} J^{\alpha}|f(s)|(\xi) \\
\leq & \frac{\psi(r)}{\Gamma(q)}\left|\int_{0}^{\tau_{1}}\left[\left(\tau_{2}-s\right)^{q-1}-\left(\tau_{1}-s\right)^{q-1}\right] p(s) d s+\int_{\tau_{1}}^{\tau_{2}}\left(\tau_{2}-s\right)^{q-1} p(s) d s\right| \\
& +\psi(r) \frac{\left|\tau_{2}-\tau_{1}\right|}{|\Lambda|}\left(J^{q} p(s)(T)+|\beta|^{\rho} I^{q} J^{q} p(s)(\xi)\right) .
\end{aligned}
$$

Obviously the right hand side of the above inequality tends to zero independently of $x \in B_{r}$ as $\tau_{2}-\tau_{1} \rightarrow 0$. Therefore it follows by the Ascoli-Arzelá theorem that $\mathcal{A}: \mathcal{C} \rightarrow \mathcal{P}(\mathcal{C})$ is completely continuous.

Next we show that $\mathcal{B}$ is an upper semi-continuous multi-valued mapping. It is known by Lemma 4.2 that $\mathcal{B}$ will be upper semicontinuous if we establish that it has a closed graph, since it is already shown to be completely continuous. Thus we will prove that $\mathcal{A}$ has a closed graph.

Claim III: $\mathcal{A}$ has a closed graph. Let $x_{n} \rightarrow x_{*}, h_{n} \in \mathcal{A}\left(x_{n}\right)$ and $h_{n} \rightarrow h_{*}$. Then we need to show that $h_{*} \in \mathcal{A}\left(x_{*}\right)$. Associated with $h_{n} \in \mathcal{A}\left(x_{n}\right)$, there exists $f_{n} \in S_{F, x_{n}}$ such that for each $t \in[0, T]$,

$$
h(t)=J^{\alpha} f_{n}(s)(t)+\frac{t}{\Lambda}\left\{\beta^{\rho} I^{q} J^{\alpha} f_{n}(s)(\xi)-J^{\alpha} f_{n}(s)(T)\right\} .
$$

Thus it suffices to show that there exists $f_{*} \in S_{F, x_{*}}$ such that for each $t \in[0, T]$

$$
h_{*}(t)=J^{\alpha} f_{*}(s)(t)+\frac{t}{\Lambda}\left\{\beta^{\rho} I^{q} J^{\alpha} f_{*}(s)(\xi)-J^{\alpha} f_{*}(s)(T)\right\} .
$$

Let us consider the linear operator $\Theta: L^{1}([0, T], \mathbb{R}) \rightarrow \mathcal{C}$ given by

$$
f \mapsto \Theta(f)(t)=J^{\alpha} f(s)(t)+\frac{t}{\Lambda}\left\{\beta^{\rho} I^{q} J^{\alpha} f(s)(\xi)-J^{\alpha} f(s)(T)\right\} .
$$

Observe that

$$
\left\|h_{n}(t)-h_{*}(t)\right\|=\| J^{\alpha}\left(f_{n}(s)-f_{*}(s)\right)(t)
$$




$$
+\frac{t}{\Lambda}\left\{\beta^{\rho} I^{q} J^{\alpha}\left(f_{n}(s)-f_{*}(s)\right)(\xi)-J^{\alpha}\left(f_{n}(s)-f_{*}(s)\right)(T)\right\} \| \rightarrow 0,
$$

as $n \rightarrow \infty$. Thus, it follows by Lemma 4.3 that $\Theta \circ S_{F}$ is a closed graph operator. Further, we have $h_{n}(t) \in \Theta\left(S_{F, x_{n}}\right)$. Since $x_{n} \rightarrow x_{*}$, we have that

$$
h_{*}(t)=J^{\alpha} f_{*}(s)(t)+\frac{t}{\Lambda}\left\{\beta^{\rho} I^{q} J^{\alpha} f_{*}(s)(\xi)-J^{\alpha} f_{*}(s)(T)\right\},
$$

for some $f_{*} \in S_{F, x_{*}}$. Hence $\mathcal{A}$ has a closed graph (and therefore has closed values). In consequence, the operator $\mathcal{A}$ is compact valued and upper semicontinuous.

Thus the operators $\mathcal{A}$ and $\mathcal{B}$ satisfy all the conditions of Theorem 4.4 and hence its conclusion implies either condition (i) or condition (ii) holds. We show that the conclusion (ii) is not possible. If $x \in \lambda \mathcal{A}(x)+\lambda \mathcal{B}(x)$ for $\lambda \in(0,1)$, then there exist $f \in S_{F, x}$ and $g \in S_{G, x}$ such that

$$
\begin{aligned}
x(t)= & J^{\alpha} f(s)(t)+\frac{t}{\Lambda}\left\{\beta^{\rho} I^{q} J^{\alpha} f(s)(\xi)-J^{\alpha} f(s)(T)\right\} \\
& +J^{\alpha} g(s)(t)+\frac{t}{\Lambda}\left\{\beta^{\rho} I^{q} J^{\alpha} g(s)(\xi)-J^{\alpha} g(s)(T)\right\}, \quad t \in[0, T] .
\end{aligned}
$$

By our assumptions, we can obtain

$$
\begin{aligned}
|x(t)| \leq & \psi(\|x\|)\left\{J^{\alpha} p(s)(T)+\frac{|\beta| T}{|\Lambda|}{ }^{\rho} I^{q} J^{\alpha} p(s)(\xi)+\frac{T}{|\Lambda|} J^{\alpha} p(s)(T)\right\} \\
& +J^{\alpha} M(s)(T)+\frac{|\beta| T}{|\Lambda|} \rho I^{q} J^{\alpha} M(s)(\xi)+\frac{T}{|\Lambda|} J^{\alpha} M(s)(T) .
\end{aligned}
$$

Thus

$$
\begin{aligned}
\|x\| & \leq \psi(\|x\|)\left\{J^{\alpha} p(s)(T)+\frac{|\beta| T}{|\Lambda|} \rho I^{q} J^{\alpha} p(s)(\xi)+\frac{T}{|\Lambda|} J^{\alpha} p(s)(T)\right\} \\
& +J^{\alpha} M(s)(T)+\frac{|\beta| T}{|\Lambda|}{ }^{\rho} I^{q} J^{\alpha} M(s)(\xi)+\frac{T}{|\Lambda|} J^{\alpha} M(s)(T) .
\end{aligned}
$$

If condition (ii) of Theorem 4.4 holds, then there exists $\lambda \in(0,1)$ and $x \in \partial B_{M}$ with $x=\lambda \mathcal{N}(x)$. Then, $x$ is a solution of (2) with $\|x\|=M$. Now, by the inequality (21), we get

$$
\frac{M}{\psi(M) \Psi_{1}+\Psi_{2}} \leq 1,
$$

which contradicts (18). Hence, $\mathcal{N}$ has a fixed point in $[0, T]$ by Theorem 4.4, and consequently the problem (2) has a solution. This completes the proof. 


\section{References}

[1] I. Podlubny, Fractional Differential Equations, Academic Press, San Diego, 1999.

[2] A.A. Kilbas, H.M. Srivastava, J. J. Trujillo, Theory and Applications of Fractional Differential Equations, North-Holland Mathematics Studies, 204. Elsevier Science B.V., Amsterdam, 2006.

[3] J. Klafter, S. C Lim, R. Metzler (Editors), Fractional Dynamics in Physics, World Scientific, Singapore, 2011.

[4] Y. Zhou, Basic theory of fractional differential equations, World Scientific Publishing Co. Pte. Ltd., Hackensack, NJ, 2014.

[5] B. Ahmad, J.J. Nieto, Riemann-Liouville fractional integro-differential equations with fractional nonlocal integral boundary conditions, Bound. Value Probl. (2011) 2011:36.

[6] Z.B. Bai, W. Sun, Existence and multiplicity of positive solutions for singular fractional boundary value problems, Comput. Math. Appl. 63 (2012), 1369-1381.

[7] D. O'Regan, S. Stanek, Fractional boundary value problems with singularities in space variables, Nonlinear Dynam. 71 (2013), 641-652.

[8] B. Ahmad, S.K. Ntouyas, A. Alsaedi, A study of nonlinear fractional differential equations of arbitrary order with Riemann-Liouville type multistrip boundary conditions, Math. Probl. Eng. Art. ID 320415, (2013), 9 pp.

[9] L. Zhang, B. Ahmad, G. Wang, R.P. Agarwal, Nonlinear fractional integro-differential equations on unbounded domains in a Banach space, J. Comput. Appl. Math. 249 (2013), 51-56.

[10] X. Liu, M. Jia, W. Ge, Multiple solutions of a p-Laplacian model involving a fractional derivative, Adv. Differ. Equ. (2013), 2013:126.

[11] J.R. Wang, Y. Zhou, M. Feckan, On the nonlocal Cauchy problem for semilinear fractional order evolution equations, Cent. Eur. J. Math. 12 (2014), 911-922.

[12] J. Henderson, R. Luca, A. Tudorache, On a system of fractional differential equations with coupled integral boundary conditions, Fract. Calc. Appl. Anal. 18 (2015), 361-386.

[13] S.K. Ntouyas, S. Etemad, On the existence of solutions for fractional differential inclusions with sum and integral boundary conditions, Appl. Math. Comput. 266 (2015), 235-243.

[14] S.K. Ntouyas, S. Etemad, J. Tariboon, Existence of solutions for fractional differential inclusions with integral boundary conditions, Bound. Value Prob. (2015), 2015:92.

[15] S.K. Ntouyas, S. Etemad, J. Tariboon, Existence results for multi-term fractional differential inclusions, Adv. Differ. Equ. (2015), 2015:140.

[16] D. Qarout, B. Ahmad, A. Alsaedi, Existence theorems for semi-linear Caputo fractional differential equations with nonlocal discrete and integral boundary conditions, Fract. Calc. Appl. Anal. 19(2016), 463479.

[17] B. Ahmad, S.K. Ntouyas, Some fractional-order one-dimensional semi-linear problems under nonlocal integral boundary conditions, Rev. R. Acad. Cienc. Exactas Fís. Nat. Ser. A Math. RACSAM 110 (2016), 159-172.

[18] M. Jia, H. Zhang, Q. Chen, Existence of positive solutions for fractional differential equation with integral boundary conditions on the half-line, Bound. Value Probl. 2016, 2016:104. 
[19] B. Ahmad, S.K. Ntouyas, J. Tariboon, A study of mixed Hadamard and RiemannLiouville fractional integro-differential inclusions via endpoint theory, Appl. Math. Lett. 52 (2016), 9-14.

[20] U.N. Katugampola, New Approach to a generalized fractional integral, Appl. Math. Comput. 218 (2015), 860-865.

[21] A.B. Malinowska, T. Odzijewicz, D.F.M. Torres, Advanced Methods in the Fractional Calculus of Variations, Springer, 2015.

[22] A.G. Butkovskii, S.S. Postnov, E.A. Postnova, Fractional integro-differential calculus and its control-theoretical applications in mathematical fundamentals and the problem of interpretation, Automation and Remote Control 74 (2013), 543-574.

[23] S. Pooseh, R. Almeida, D.F.M. Torres, Expansion formulas in terms of integer-order derivatives for the Hadamard fractional integral and derivative, Numer. Funct. Anal. Optim. 33 (2012), 301-319.

[24] T.A. Burton, C. Kirk, A fixed point theorem of Krasnoselskii-Schaefer type, Math. Nachr. 189 (1998), 23-31.

[25] W.V. Petryshyn, P.M. Fitzpatric, A degree theory, fixed point theorems, and mapping theorems for multivalued noncompact maps, Trans. Amer. Math. Soc., 194 (1974), 1-25.

[26] K. Deimling, Multivalued Differential Equations, Walter De Gruyter, Berlin-New York, 1992.

[27] Sh. Hu, N. Papageorgiou, Handbook of Multivalued Analysis, Volume I: Theory, Kluwer, Dordrecht, 1997.

[28] G.V. Smirnov, Introduction to the theory of differential inclusions, American Mathematical Society, Providence, RI, 2002.

[29] A. Lasota, Z. Opial, An application of the Kakutani-Ky Fan theorem in the theory of ordinary differential equations, Bull. Acad. Polon. Sci. Ser.Sci. Math. Astronom. Phys. 13 (1965), 781-786.

[30] C. Castaing, M. Valadier, Convex Analysis and Measurable Multifunctions, Lecture Notes in Mathematics 580, Springer-Verlag, Berlin-Heidelberg-New York, 1977. 